\title{
PERSONAL CONTRIBUTIONS REGARDING SELECTION, IDENTIFICATION AND PROMOTION OF TALENTS IN FOOTBALL
}

\author{
Arben KAÇURRI ${ }^{*}$ Irakli QURKU² \\ ${ }^{1}$ Sports University of Tirana, Institute of Sport Research, Tirana, Albania \\ ${ }^{2}$ ACS Gloria Kids, Bucharest, Romania \\ *Corresponding author: akacurri@ust.edu.al
}

DOI: https://doi.org/10.51267/icpesk2020bp06

\begin{abstract}
The term "selection" has provoked disputes among various people, some of whom, for institutional reasons, defending the cause of a sport that enjoys great popularity and aims to attract all possible talents on the football field. From our point of view, football selection is not a unique, singular action. It has a lasting character and is carried out in several stages. The efficiency of the selection greatly depends on the quality of the training process and is largely conditioned by the way in which the particularities of growth and development of children and juniors are taken into account. By using the scientific selection process in football, the path to achieving remarkable (often incredible) performance becomes shorter. The selected individual undergoes a careful training process and tries to obtain maximum efficiency in the shortest time (depending on the biological potential). Also, the purpose of this paper is to improve the duration of the educational and training process, based on the improvement of the selection process correlated with the sports training stages in the football game. Selection and training have to be regarded according to the following training-type models: children, juniors III, juniors II, juniors I, which must take into account the criteria: health status, social condition, physical ability, body size, general motor skills, favourable motor skills, psychological ability, technical and tactical training, and game testing. We believe that, by contributing to the knowledge of the above-mentioned particularities, this paper eliminates the possibility of methodological errors that can have irreversible negative consequences.
\end{abstract}

Keywords: selection, talent, football, training.

\section{Introduction}

"Football is one of the most popular sports in the world. Professional football has become a significant contributor to global economics and business. The game attracts considerable funds, which motivate participants of the sporting process (players, coaches, club owners, administration, etc.) to strive for better athletic results." (Qader et al., 2017, p. 38)

Selection is a task of particular importance in all fields of activity, especially in those where the competitive element predominates. The motto "the right man at the right place" becomes an imperative nowadays and expresses the concern to "match" human skills with the demands of an activity. For many millennia, the only criterion of selection was the activity itself.

In time, this was defined as the uninterrupted process of finding, choosing and sorting young people according to certain well-defined criteria and guiding them towards the organized practice of a sporting event or field, in which they can use their qualities to the highest degree in order to obtain superior performance.

Performance behaviour depends on showing the structure in its forms and the nature and characteristics of the stimulus that causes it. Moreover, it also encompasses the particularities of athletes. (Stroescu, 2017) 
Selection is therefore the process by which people with talent and sport-friendly capabilities are individualised using scientific methods and tests.

According to Jiménez and Pain (2008), "professional and national team players show a behaviour that weakens with ageing, which suggests that talent identification and selection processes can be improved to help better identify potential talent early on and minimise wasted potential" (p. 995).

The efficiency of the selection greatly depends on the quality of the training process, which is largely conditioned by the way in which the particularities of growth and development of children and juniors are taken into account.

Another important aspect is the coach's knowledge of individual motor skills and abilities of each athlete and placing them on a scale needed to achieve the desired performance level (Stroescu, 2011). These relate to individual characteristics that are more or less significant in the same age period because the level of development indices can vary within quite wide limits. The individual treatment of the child as well as the respect of age particularities are a necessity for effectively organizing and leading the training process in the football game, but also for training and developing the personality of each young person.

We can see how important the selection action is for the individual, specialists (coaches, doctors, psychologists) and society. We consider that, in addition to the conceptual progress of the selection, the interrelation between the following elements makes up this activity:

- permanent, constant, gradual, organized, repeated process;

- $\quad$ systematic and responsible activity based on scientific methods;

- finding, choosing, sorting - early identification of children, adolescents, young people, individuals;

- well-defined criteria through the knowledge by specialists (teachers, coaches, doctors, psychologists, etc.) of the favourable skills for sport, such as talent and laws of somatic, motor, and psychological growth;

- specific requirements for performance sport through specific and scientific training in a certain sports branch;

- obtaining superior performance.

\section{Problem statement}

Nowadays, selection is a very important issue in sports for several reasons:

- performance has reached a very high level;

- rigorous observance of particularities in the practice of high-performance sports (constant and long-term training, use of large loads, many repetitions, maximum functional demands, etc.);

- worldwide assertion of the sporting qualities of a nation (in our case, Romanian athletes and Romanian results).

The timeliness of the research undertaken arises from the need to improve the duration of the educational process, based on the improvement of the selection process correlated with the stages of sports training in the football game. In this context, both the quantity and quality of the educational and training process in children and young people are a decisive factor in 
ensuring that players can perform better in the future, in line with the current game and its spectacular evolution (total commitment and ignoring strict specialisation in positions).

We should not forget that, in parallel with the selection, we need to follow the training plans that ensure continuity and optimal training. "They can provide information on the dynamics of the evolution of performance capacity, the structures or systems of action exercises, methods used in the process of training volume and intensity, duration of pauses, their nature, estimated amount of claims, evaluation forms and their dates, duration of each component" (Stroescu, 2014, p. 243).

By using the scientific selection process in football, the selected individual undergoes a carefully guided training process and tries to achieve maximum efficiency in the shortest time (depending on their biological potential).

However, what happens when, after this complex selection process, we notice that an individual is not talented for football but is equipped with general motor skills? Is it serious that such an individual has been involved in this complex process? Is it wasted time?

No, because in this case, the new concept of "orientation and selection" comes into play, being largely outlined internationally, and applied by us at different times.

\section{Purpose of study}

Regarding the selection in football, we can say that it is a very broad topic but very briefly addressed in the literature as a concept. In light of these theoretical considerations, the novelty of this study consists in creating a guide based on solving the main questions that arise in the selection process.

We consider that the issue of selection is particularly important to identify and promote "talents" in football and, through this paper, we try to address this topic in accordance with all the requirements of training science.

Also, the purpose of this work is to improve the duration of the educational process, based on the improvement of the selection process correlated with the stages of sports training in the football game.

Nowadays, football specialists are thinking more and more about creating a "pyramid" that will be based on selection, with the great performer at the top. Therefore, we attach the highest importance to the main criteria for the selection and training of children and juniors, which ensure the exchange of generations in tomorrow's football.

\section{Research methods}

As research methods, we used both an extensive theoretical review to highlight the main qualities pursued in the selection of future football players and, at the same time, our experience as coaches and football players.

All the theoretical data used to achieve this article present various studies conducted over several years and a thorough analysis of the importance of selection. 


\section{Topic Addressed}

\section{Selection in football}

From our point of view, football selection is not a unique, singular action. It has a lasting character and is carried out in several stages. The efficiency of the selection greatly depends on the quality of the training process and is largely conditioned by the way in which the particularities of growth and development of children and juniors are taken into account.

Each stage of growth and development of the human body has specific rules dictated by the particularities of age and gender, which have to be very well known by the coach in order to carry out a scientific direction of training. Not knowing or ignoring them leads to the training of children and juniors according to training schemes used in adult athletes. Thus, great harm is brought to both the health of young athletes and the training of valuable and competitive players.

In this context, we should not confuse chronological age (effective age in years and months) with biological age (assessed based on the actual level of the "morphological" and "physiological" functional somatic indices, which can place the individual in the normal chronological age category or in another younger or older one).

A study conducted on 202 players in the U9 to U16 age groups over an eight-year period showed that their relative age (birth quarter) and biological maturity (based on the KhamisRoche method of percentage of predicted adult height at the time of observation) were categorised as late maturing (Hill et al., 2019).

\section{The optimum age of selection in football}

Football can be practised from the young age of 6-10 to the age of 37-40, which is why improving the sporting outcome requires the knowledge of all particularities of these age stages.

As for determining the optimal age of selection in football, there are still conflicting views. Each of the existing concepts and practices is presented with their advantages, but practice has shown that there is a certain optimal selection period. We consider as the first selection of children the initiation phase at the age of 7 , followed by the second phase at the age of 11 when we can more clearly distinguish the individual potential for performance, and the third phase, Juniors I, at 17 years when we notice their maturity and playing abilities.

Achieving high-level performance at a very young age with the lowest possible material costs is the ideal for any coach or sports manager. With the value of sports performance rising to very high levels to be at the top of world rankings, the factors leading to such performance need to be as precise as possible:

- the human body,

- methodical training. 
For children at a start-up level, the training process will be conducted with technical exercises aimed at multilaterally training the body and providing the basis for special training. Proper learning of special technical procedures is a guarantee that performance is achieved.

In children, sport is also related to the number of years needed to learn specific processes and get through morphological changes at puberty. This period (difficult for sports in general) can sometimes occur brutally and can change the somatic biotype so that the athlete is no longer able to cope with the demands of the sport. In order for this critical moment to be overcome and for the child's growth and development to resume its normal biological course, a good coach-physician-athlete collaboration is necessary.

Medical and sports selection criteria have long been part of the arsenal of theoretical knowledge. However, the problem arises in their practical application, which is not always done correctly and efficiently. Puberty, in addition to morphophysiological changes and sexual maturation, also induces neuropsychological changes. Accelerated sexual development and neoteny are two phenomena characteristic of our half century and need to be recalled in this context because they are important for the optimal age of selection in sport. "Biological maturity status significantly influences the functional capacity of adolescent football players 13-15 years of age" (Malina et al., 2004, p. 555).

In terms of cardiovascular system, the detection of real or apparent cardiovascular pathological aspects in selected athletes is a priority objective aiming to eliminate both the causes that may limit biological performance skills and subjects with a potentially high risk to health and life.

Absolute cardiovascular contraindications in primary selection are: aortic values (especially aortic stenosis), mitral valve prolapse with regurgitation and arrhythmias, pre-excitation syndromes with arrhythmias; relative contraindications are: interatrial septal defect, preexcitation syndromes without rhythm disturbances, interventricular septal defect, mitosis.

Absolute cardiovascular contraindications in secondary and final selections are: hypertrophic cardiomyopathy, mitral and aortic valve, mitral valve prolapse with regurgitation and rhythm disorders, interventricular septal defect, pre-excitation syndromes with rhythm disorders, secondary hypertension; relative contraindications are: clinically well-tolerated interatrial septal defect and no load of pulmonary circulation, some rhythm disorders (relatively rare non-systematised extra-systole that disappears during exercise).

To explore the cardiovascular system, specific effort tests are used, after which different indices are recorded (pulse, blood pressure, time to return to normal). Standard efforts can also be used, the most important being: Martinet test, EKG, oximetry, haemodynamic examination. Telemetry results also support injury statistics by indicating that aerobic conditioning should have a significant role in the training programme (Gleim et al., 1981).

For sports medicine, cardiovascular assistance begins with primary selection and usually ends when competitive activity is completed. It is ideal, if possible, to set up the process of tracking former athletes after the end of their performance careers, which would complement the understanding of the effects beyond physical performance effort.

In the selection process, we will take into account the pathology of the respiratory system, which includes elements related to the chest, airways, lung parenchyma, which affect respiratory mechanics and gas diffusion. 
Clinical aspects are: laryngeal spasm, spasms, tetanus, acute laryngeal oedema, allergy, infectious diseases, inflammation of the respiratory tract and parenchyma, a tendency to asthma, chocking. "Prevention of illnesses should focus on reducing the risk of infections by considering the common modes of transmission and environmental conditions" (Dvorak et al., 2011, p. 626).

The neuromuscular system can be controlled through methods such as chronoximetry, electromyography, or dynamometry. The functionality of central and peripheral nervous systems is closely related to the muscular activity, which is much involved in performing the motor act. "A protective effect on injury risk in youth sports through neuromuscular warm-up training routines has consistently been demonstrated. However, there is a paucity of information regarding the quantity and quality of coach-led injury prevention programmes and its impact on the physical performance of players." (Steffen et al., 2013, p. 794)

In the secondary and final selections, with the help of sports medicine, we can assess whether the training causes changes in neuromuscular excitability (lower values of galvanic intensity of the nerve and higher values of the muscle).

\section{General considerations regarding the detection of football talents}

Talent selection is a stepping-stone to sport success at national and international levels (Brad \& Popi, 2016).

According to Dina (2012, p. 48), talent results from the interrelation between endogenous factors (anthropometric characteristics, motor ability, psychological factors, physiological and functional apparatus) and exogenous factors (training and ambiance).

Talent is defined by Nadori (1993) as an ability or a group of abilities with a certain specificity that is well above average and has to be expressed. It develops during the practice of sport and needs the best conditions to be highlighted.

Talent in football encompasses the top level of initial performance, the high rate of performance growth, and the easy acquisition of theoretical and practical knowledge.

Talent has a number of basic features, among which we emphasise:

- more efficient reaction to stimuli and especially to high-intensity stimuli;

- correct and creative application of techniques;

- better use of it by specifically combining simple or complex abilities, skills, attitudes and directions of interest;

- a developed kinaesthetic sense.

One of the latest directions in talent research is the genetic line that includes:

- research of the family tree;

- the ratio between the motor performance of parents and that of children;

- studies performed on twins of both genders;

- studies of hereditary transmitted characteristics.

Among the results obtained from the research of these directions, we can mention:

- $50 \%$ of children with special sports skills have parents who were good athletes (Strakosha Thomas - Lazio Roma team);

- the sport practiced by parents may be different from the one practiced by their children; 
- homozygous twins have more common characteristics than heterozygous ones;

- the extent and overall possibility of increasing performance are apparently limited by genetic factors.

But the issue of gifted children is also topical.

The six areas of manifestation of gifted children's performance include psychomotor skills (along with general intellectual ability, exceptional academic ability of creative or productive thinking, leadership skills, talent for visual and performing arts).

We outline a theoretical model for future football talents.

1. General and specific intellectual abilities:

- abstract thinking;

- integration of experiences;

2. High-level motivational commitment accompanied by courage, perseverance, tenacity, patience;

3. Exceptional skills that ensure special value "responses";

4. Creativity (fluency, flexibility, originality, receptivity to the new, curiosity, adventure, risk-taking);

5. The courage to try one's own chances;

6. The desire to be a gifted child;

7. The need for excellence - the desire for supremacy;

8. The level of psychomotor skills supported from a motivational, attitudinal (I want, I can), emotional, cognitive point of view and in a positive relationship with intellectual development;

9. The longing for extraordinary performance is close to heroism and self-forgetfulness.

\section{Dynamics of growth and development of physical and psychological skills in football}

A child must receive many opportunities to have motor experiences and improve motor coordination in order to develop successfully motor skills (Alesi et al., 2015). The qualities of the young players are tested during the activity, therefore constant participation in the training is the most important external condition for identifying sports skills.

As regards sporting talent, it is useful to establish very early, at the moment of technical maturity of the subjects, if they have a promising future. Baker et al. (2019) propose a multifaceted conceptualisation of talent as: innate (i.e. originating in biological elements present at birth), multidimensional (i.e. consisting of abilities from a range of broad cognitive, physical and psychological categories), emerging (i.e. involving interactions among factors that combine multiplicatively), dynamic (i.e. evolving across developmental time due to interactions with environments and random gene expression) and symbiotic (i.e. cultural and social factors will determine the ultimate value of an individual's talent). Simplifying, we can define football talent as a special psycho-physical condition and an activity that goes through a certain evolution, so that it exceeds the average.

For various reasons, this is important for the coach, for the various categories of club's technical staff, as well as for (other) clubs that intend to invest in certain young players using their own infrastructure. 
Also, it is necessary to know how to identify athletes who need a specific method or training to improve the level of skills that they naturally possess.

A motor skill is a learned sequence of movements that combine to produce a smooth, efficient action in order to become expert in specific goal-directed tasks (Farhat et al., 2015). The improvement of motor and cognitive abilities is related not only to general physical activity but also to specific ability related to the ball (Alesi et al., 2015).

A common question is: "Which age group should pay more attention to identifying potential talents and which factor is most significant?"

A child can have a favourable genetic background (hereditary factors) as well as a certain experience, even if it is minimal (environmental factors). It is very clear that if nothing can be done to influence the first category of factors, there are many things that can be done to learn the specific elements.

For example, a sprinter's son is likely to have the same muscle qualities as his father (\% fast fibres). However, if certain features are not prompted through specific training and development programmes, their potential may remain partially undiscovered (latent).

Another important aspect is that, in order to identify a potential talent, it is necessary to analyse not only the overall activity (efficiency) but also the factors intrinsic to performance.

According to Williams and Reilly (2000), "research approaches in anthropometry, physiology, psychology and sociology are considered and, where possible, integrated. Although some progress has been made in identifying correlates of playing success, it appears that no unique characteristics can be isolated with confidence. Both biological and behaviour scientists have indicated a strong genetic component in performance of sports such as soccer; nevertheless, the influence of systematic training and development programmes should not be underestimated" (p. 657).

Experience has shown that sometimes, regardless of whether young players were selected on the basis of hereditary or acquired elements and whether their further promotion (differentiated training within high-level teams) was carried out according to the most appropriate modern training methods, certain potential talents do not reach the expected results. This means that there may have been some misinterpretation of the various indicators used for talent assessment or that the indicators used have not been sufficiently stable over time.

Another possibility is that the psychological, motivational and social factors have had a negative impact on the athlete's technical career. However, the opposite situation was recorded, for example, some players were not selected as talents at a young age but evolved and reached professional categories. This means that there is an $\mathrm{x}$ factor that has not yet been discovered or that talent identification is not an absolute science.

In a study (Gledhill et al., 2017) investigating 14,977 participants and 48 psychosocial factors associated with talent development in football, the findings suggest that social and psychological factors are interrelated and influence adaptive developmental behaviours (e.g. adaptive lifestyle choices, practice and play behaviours). These can influence coach perceptions of players and whether they may recommend players for career progression.

Figure 1 shows the identification plan for talented football players, and Figure 2, the parameters that can be used to evaluate a football player. 


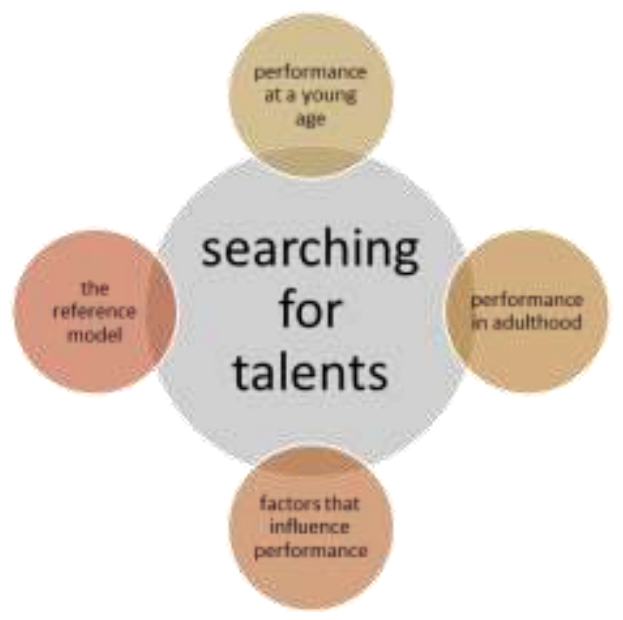

Figure 1. Identification plan for talented football players
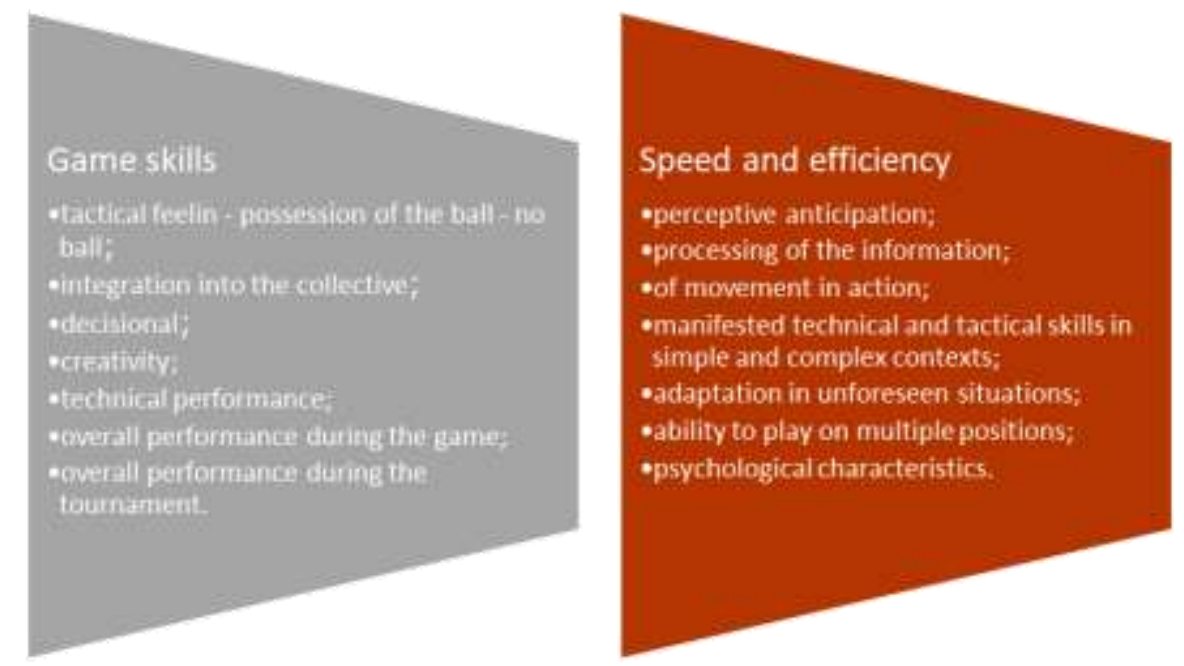

Figure 2. Parameters that can be used to evaluate a football player

At the age of 7-8, we can see certain skills that can give the image of a potential football talent. Even if it is too early for a child to be classified as such, it may be that their performance level is higher than that of children in their age group. In general, "promises" are more easily fulfilled if the overall picture of the factors that define the sporting activity has a significant potential for improvement. At the age of 12-13 years, a first selection (prediction) can be made, but the activity in the coming years and the promotion process will show whether or not these pre-existing conditions are maintained over time (at least 3-4 years).

In other words, for the promotion of talents, it is important to select an appropriate prognosis method. We conclude that sport and exercise sciences play an important supportive role in the processes of identifying, monitoring, and nurturing talented football players towards achieving their potential. 


\section{Key stages in the development of physical and psychological skills in children}

In any sports discipline, it is essential for coaches to have as precise and detailed data as possible about the needs of athletes and the efficiency of methods used to improve the learning process (Grigoroiu et al., 2019). The teaching/learning process depends on the relationship between coach and athlete and develops based on the correspondence between the stimulus and the response to it.

In applying the teaching incentives, the order of exercises and specific techniques, the child's emotional ability and developmental stages should be taken into account, even if they differ from individual to individual and the biological age does not always correspond to the actual age of the athlete. In this context, one of the extremely important tasks for the coach is to closely monitor the development of students and offer them what they "can" learn at the "right time".

"Based on recent, dynamic-interactionist concepts of development, we assume a personoriented approach focusing on the person as a whole rather than individual features. Theoretical considerations lead to four interacting factors being summarised to form a subsystem: childhood training. The relative weights of these factors lead to patterns. By relating these to a performance criterion at the age of peak performance, particularly promising developmental patterns may be identified." (Zibung \& Conzelmann, 2013, p. 452)

The favourable period in which a "development peak" of the coordination abilities can be observed is between 7 and 12 years old. It seems that speed develops in the same period with the ability to react and especially the frequency of movement, while the acyclic speed and the speed of action reach the maximum level a few years later.

The pace of development decreases after this stage, and adaptation stimuli no longer produce adequate responses. In this sense, training methods that favour the general development of motor skills should be used by expanding the range of situations and multifunctional activities from several sports.

Currently, elite football requires strong and tough athletes with superior motor and functional skills (speed, explosive strength, aerobic and anaerobic capacity, coordination), and a sense of improvisation and team play. "A skilled player who understands tactics will never be able to show what he is capable of when he rarely gets the ball due to bad physical condition, moves slowly across the pitch, and strikes the ball poorly. Muscle strength and speed allow the player to use his entire technical and tactical arsenal in a game." (Bolotin \& Bakayev, 2017, p. 406)

A key stage in the development of speed-strength and strength-endurance is around the age of 9. It is important to reduce external resistance so that the contraction speed can be increased (it is recommended to use natural weights).

Strength and endurance are important co-determinants of football performance, as they are key features of physical ability and main regulators of important football-specific tasks (Hoff \& Helgerud, 2004).

Maximum strength training begins in the first period of puberty. Aerobic endurance is a developmentally neutral ability, with the peak of growth being observed at puberty. In contrast, it is relatively difficult to train anaerobic endurance due to both biological limitations 
(insufficient testosterone secretion correlated with certain anaerobic glycolysis enzymes) and the psychological resilience required for certain activities.

It is very important to respect the model of key stages presented in the following lines:

1. Psychological and motor functions - coordination skills:

- ability to learn motor movements (begins to develop uniformly from the age of 7 to 10 , after which there is an increase between 10-12 years old, stagnation between the ages of 12-14, and the learning ability can be developed again at the age of 14 to 15);

- ability to differentiate and control (starts to grow from the age of 7 to 10 , increases at 10-12 years, stagnates between the ages of 12-14 and is moderate at 14 to 15 years);

- ability to react to optical and acoustic stimuli (begins to grow from the age of 7 , significantly increases between 8 and 10 years old, then decreases and ends at the age of 11);

- $\quad$ spatial orientation ability (starts from the age of 6 years, constantly develops up to 12 years, then increases until the age of 15);

- ability to maintain balance (develops between 9 and 13 years old, with a higher increase between the ages of 10 and 12).

2. Physical abilities:

- endurance (can be developed between the ages of 6-11 and, starting with the age of 12, can be developed at maximum parameters);

- strength (can be developed between the ages of 8-12 and, starting with the age of 12, can be developed at maximum parameters);

- $\quad$ speed (must be trained at maximum parameters between the ages of 7-12, after which it is maintained).

3. Emotional and cognitive abilities:

- emotional and cognitive skills (develop in stages starting with the age of 7 , the favourable development period being between the ages of 10-12 years);

- willingness to learn (starts from the age of 6 to 13, with an increase between 7 and 11).

Guide to the main aspects of evaluating the selection process and the methods to improve it

Winning matches or using the selection process by age group are simple units of measurement. These skills are easy to measure, but are they real indicators of a successful development process?

Consider the following examples:

When measuring the degree of learning, this is often done by setting an indicator to compare the acquired knowledge with the previous one. This indicator is useless when the player, even if he has accumulated theoretical knowledge, is unable to express it on the field. This algorithm can also be applied to the development process.

It is very important, first of all, to have a set of indicators to follow during the selection process. They must be used in the actual measurement of the system, taking into account the complexity of this process.

Aspects of evaluation can be found to be objective (they are easier to collect) or subjective (they can be just as effective in the evaluation process). 
Objective evaluations:

- Number of players from the first team of the club;

- Number of players in the senior team;

- Number of players promoted to the first team;

- Number of championships and tournaments played;

- Number of matches: lost, won, drawn;

- Total number of players under preparation;

- Experience gained in the activity;

- Number of training hours;

- The cost of these hours;

- Market value of players, etc.

Progress is ensured by two factors:

1. Selection will be made taking into account the value and football skills of the players, even at a young age.

2. Developing solid opinions regarding the player's development potential within the standards set by the club!

The purpose and objectives of talent identification are:

1. To identify valuable players who are able to compete and be successful, who demonstrate that they are physically and psychologically prepared to meet the requirements of the game (this point is used for immediate purposes in winning matches).

2. To identify players who promise but have physical and technical difficulties (this point is used for long-term development purposes and to identify players who need special attention from the coach and the observer).

The individual quality that the player demonstrates will be the most important way to successfully evaluate the programme. Progress will be tracked and analysed over a wellestablished period of time. But what is this period of 1 year, 3 years, 5 years, 10 years?

Personal experience has shown us that there are three factors that, if not properly controlled, can compromise the entire preparation process:

A 10-year training cycle is commonly used. The results can be influenced by:

- The atmosphere created by the coach;

- The geographical and cultural areas of the club and players;

- Expectations of the club management.

Proposed methods for improving the selection process:

- Maximising the potential of the players;

- Identifying the player's evolving quality within the club's requirements;

- In football terms, a young and successful coach who has a realistic development programme will regularly prepare players for the first team;

- Quality standards established by the club are those that establish the quality of training;

- These usually high standards limit the number of truly competent players.

Question: What is the "quality for football" of the evaluated player?

Answer - Subjective evaluations:

- Opinions about the coach will reflect the qualities of the players;

- All evaluations will have to be demonstrated to the players;

- The subjectivism of the coaches regarding the abilities and potential of the players; 
- Comparisons between players of the same age;

- Comparisons between different stages of evolution of the same player.

Question: How can you measure evolution if the position in the ranking changes after the evaluation process?

By identifying and evaluating the players' current qualities and ability to progress. If the first team is to be improved, identifying true talents is essential for players to develop the club's standard.

Question: Do players have the potential for development? If so, how much and where?

The biological model of the performer varies from one stage to another, it is constantly changing. By individualising a talented person with the help of the selection process (based on scientific methods and tests), we can bring that person to the biological model of the performer only through a carefully guided and long-term preparation process.

The objective circumstances in which the teaching process and the assimilation of knowledge occur represent the essential factor in developing the potential of the players.

Question: Can the coach understand the changes that are characteristic of aging and can the coach's knowledge influence the players' expectations?

Yes, by classifying the teams by age group.

It is more difficult to collaborate with younger players during matches or training camps because they have a fluctuating and confused behaviour but, most of the times, the expectations regarding the first group of selectors are in direct correspondence with the age of the players. Once they are chosen, coaches adopt or create a vocabulary to identify the players that can be unique to their football team.

\section{Conclusion}

The road to achieving extraordinary performance is possible today only through scientific selection determined by three factors:

- genotypic factors (related to heredity, talent);

- phenotypic factors (related to the accumulated characteristics: somatic, functional, motor and psychological);

- integrating social and sports-related factors (organization and management).

Evaluation and measurement using tests and assessments can be considered as a comparison of the plan with the result, so that corrections, reorientations and restructuring of the system can be carried out objectively with the purpose of optimising the training process and selection.

For the football game, tests and assessments are needed to check the qualities of future performance players. Without these, the selection and training processes are out of control.

Analysis of the modern game for each age category and training stage has led to a series of specific features that those involved (coaches, teachers) should take into account, considering selection (correlated with the stages of preparation) as an important component of training.

A competent and consistent specialist can exploit the individual biological potential at each stage of preparation and, following the scientific selection process, can create another (more efficient) sample model. 
Coordinated choice must be a complex framework that will work with objective (natural, therapeutic, sociological, mental, motor and systematic) markers, seeking to achieve a complex potential that, under the impact of logical training, will lead to high performance (to be predictive).

Introductory models into the football choice should be operational instruments that will incorporate functional-motor-psychological inclinations, counting tests and performance standards specific to the training stages decided by age.

This review highlights the need for coaches and researchers to consider the players' technical and tactical skills combined with their age-related anthropometric and physiological characteristics. "Moreover, research addressing the psychological and environmental aspects that influence talent identification and development in football is currently lacking" (Sarmento et al., 2018, p. 907).

In our constant search for ways to improve results, we have established that selection is one of the enabling factors that contribute not only to achieving higher sports results and accelerating their achievement, but especially to their quality as a permanent trend.

\section{Acknowledgements}

All authors have equally contributed to this study and should be considered as main authors.

\section{References}

Alesi, M., Bianco, A., Padulo, J., Luppina, G., Petrucci, M., Paoli, A., \& Pepi, A. (2015). Motor and cognitive growth following a football training program. Frontiers in Psychology, 6: 1627. https://dx.doi.org/10.3389\%2Ffpsyg.2015.01627

Baker, J., Wattie, N., \& Schorer, J. (2019). A proposed conceptualization of talent in sport: The first step in a long and winding road. Psychology of Sport and Exercise, 43, 27-33. https://doi.org/10.1016/j.psychsport.2018.12.016

Bolotin, A., \& Bakayev, V. (2017). Pedagogical conditions necessary for effective speedstrength training of young football players (15-17 years old). Journal of Human Sport and Exercise, 12(2), 405-413. doi: 10.14198/jhse.2017.122.17

Brad, H. \& Popi, S. (2016). Coach decision-making and the relative age effect on talent selection in football. European Sport Management Quarterly, 16(3), 292-315. https://doi.org/10.1080/16184742.2015.1131730

Dina, L. (2012). Selecția în Gimnastica Artistică. [Selection in Artistic Gymnastics] Craiova: Sitech.

Dvorak, J., Junge, A., Derman, W., \& Schwellnus, M. (2011). Injuries and illnesses of football players during the 2010 FIFA World Cup. British Journal of Sports Medicine, 45(8), 626630. https://doi.org/10.1136/bjsm.2010.079905

Farhat, F., Hsairi, I., Baiti, H., Cairney, J., Mchirgui, R., Masmoudi, K., Padulo, J., Triki, C., $\&$ Moalla, W. (2015). Assessment of physical fitness and exercise tolerance in children with developmental coordination disorder. Research in Developmental Disabilities, 45-46, 210 219. https://doi.org/10.1016/j.ridd.2015.07.023

Gledhill, A., Harwood, C., \& Forsdyke, D. (2017). Psychosocial factors associated with talent development in football: A systematic review. Psychology of Sport and Exercise, 31, 93112. https://doi.org/10.1016/j.psychsport.2017.04.002 
Gleim, G. W., Witman, P. A., \& Nicholas, J. A. (1981). Indirect assessment of cardiovascular "demands" using telemetry on professional football players. The American Journal of Sports Medicine, 9(3), 178-183. https://doi.org/10.1177/036354658100900309

Grigoroiu, C., Pelin, R. A., Pricop, A. D., \& Netolitzchi, M. (2019). Study on the efficiency of the myotest device in the development of the mini soccer players explosive strength. In I. Roceanu (Ed.), Proceedings of $15^{\text {th }}$ International Conference eLearning and Software for Education, "New Technologies and Redesigning Learning Spaces" (Vol. 3, pp. 475-482). Bucharest: Carol I NDU Publishing House. DOI: 10.12753/2066-026X-19-204

Hill, M., Scott, S., Malina, R. M., McGee, D., \& Cumming, S. P. (2019). Relative age and maturation selection biases in academy football. Journal of Sports Sciences, 38(11-12), 1359-1367. https://doi.org/10.1080/02640414.2019.1649524

Hoff, J., \& Helgerud, J. (2004). Endurance and strength training for soccer players: Physiological considerations. Sports Medicine, 34(3), 165-180. https://doi.org/10.2165/00007256-200434030-00003

Jiménez, I. P., \& Pain, M. T. G. (2008). Relative age effect in Spanish association football: Its extent and implications for wasted potential. Journal of Sports Sciences, 26(10), 995-1003. https://doi.org/10.1080/02640410801910285

Malina, R. M., Eisenmann, J. C., Cumming, S. P., Ribeiro, B., \& Aroso, J. (2004). Maturityassociated variation in the growth and functional capacities of youth football (soccer) players 13-15 years. European Journal of Applied Physiology, 91(5-6), 555-562. https://doi.org/10.1007/s00421-003-0995-z

Nadori, L. (1993). Talentul și selecția acestuia. [Talent and its selection]. București: CCPS.

Qader, M. A., Zaidan, B. B., Zaidan, A. A., Ali, S. K., Kamaluddin, M. A., \& Radzi, W. B. (2017). A methodology for football players selection problem based on multi-measurements criteria analysis. Measurement, 111, 38-50. https://doi.org/10.1016/j.measurement.2017.07.024

Sarmento, H., Anguera, M. T., Pereira, A., \& Araújo, D. (2018). Talent identification and development in male football: A systematic review. Sports Medicine, 48(4), 907-931. https://doi.org/10.1007/s40279-017-0851-7

Steffen, K., Emery, C. A., Romiti, M., Kang, J., Bizzini, M., Dvorak, J., \& Meeuwisse, W. H. (2013). High adherence to a neuromuscular injury prevention programme (FIFA 11+) improves functional balance and reduces injury risk in Canadian youth female football players: A cluster randomised trial. British Journal of Sports Medicine, 47(12), 794-802. https://doi.org/10.1136/bjsports-2012-091886

Stroescu, S. A. (2017). Algorithmic program - Support in learning of 'handspring forward with $1 \frac{1}{2}$ tucked salto forward' on vault. Global Journal of Guidance and Counseling in Schools: Current Perspectives, 7(1), 7-14. https://doi.org/10.18844/gjgc.v7i1.2942

Stroescu, S. A. (2011). Program for learning element "Jump backward (Flic-Flack take off) with $1 / 2$ twist $\left(180^{\circ}\right)$ through handstand to walk over forward" ("Mostepanova") on beam: Biomechanical analysis. Discobolul, 26(4), 86-93.

http://discobolulunefs.ro/Reviste/2011/Discobolul_4_2011_extenso.pdf

Stroescu, S. A. (2014). Qualitative and quantitative analysis of preparation and high-level competitions in Artistic Gymnastics between 2001 and 2008 in Romania. Procedia - Social and Behavioral Sciences, 117, 243-248. https://doi.org/10.1016/j.sbspro.2014.02.208

Williams, A. M., \& Reilly, T. (2000). Talent identification and development in soccer. Journal of Sports Sciences, 18(9), 657-667. https://doi.org/10.1080/02640410050120041

Zibung, M., \& Conzelmann, A. (2013). The role of specialisation in the promotion of young football talents: A person-oriented study. European Journal of Sport Science, 13(5), 452460. https://doi.org/10.1080/02640410050120041 